\title{
The Multi-Mission Maximum Likelihood framework (3ML)
}

\author{
Giacomo Vianello* \\ HEPL, Stanford University \\ E-mail: giacomovestanford.edu
}

\section{R. J. Lauer}

J. M. Burgess

H. Ayala

P. Harding

M. Hui

H. Zhou

\section{Savchenko}

\section{H. Fleischhack}

\section{S. Marinelli}

The age of multi-wavelength and multi-messenger astronomy has arrived and with it, new tools are needed to analyze data from multiple instruments properly and with ease. The Multi-Mission Maximum Likelihood framework (3ML) provides this functionality via the novel use of instrument plugins which allow for every instrumentâÁŹs unique data to be treated independently with an appropriate likelihood. Under the 3ML framework, users can design plugins that handle instrument specific data routines transparently in the background. When multiple instruments are used together, their independent likelihoods are treated under a common minimization or Bayesian sampling framework. 3ML provides a multitude of minimization algorithm for maximum likelihood estimation (MLE) as well as several popular Bayesian posterior samplers. The entire framework is provided via a modern Python interface providing the user with a modern and easily transportable analysis framework well suited for modern astronomy. New models can be added easily. It is also possible to perform time-energy modeling. We present the framework and its main functionalities.

7th Fermi Symposium 2017

15-20 October 2017

Garmisch-Partenkirchen, Germany

\footnotetext{
* Speaker.
} 